\title{
El papel de la Educación Física escolar en el siglo xxi: una mirada desde el docente universitario
}

\author{
Alberto Morona Doña* \\ Carmen Trigueros Cervantes ${ }^{* *}$ \\ Enrique Rivera Garcia ***
}

\begin{abstract}
Resumen: En este trabajo se analizan, cualitativamente, las percepciones de 28 docentes universitarios en relación a las funciones de la Educación Física Escolar, profundizando en la función de transformación de las desigualdades de origen. Los procedimientos de análisis empleados, en general se ciñeron a las propuestas de fragmentación y articulación de la "Grounded Theory". Se concluye que la función de transformación social de la Educación Física está relacionada con un deber ser profesional que aún está por concretarse y se establecen una serie de principios pedagógicos centrados en la necesidad de revisar el sustento epistemológico de la misma.
\end{abstract}

Palabras clave: Educación Física. Rol profesional. Responsabilidad social.

\section{INTRODUCCIÓN}

En la transformación del modelo pedagógico de la Educación Chilena, parece que la visión tecnocrática de la Educación Física (en adelante EF) está dejando paso a una perspectiva más compleja y democrática de la asignatura. Desde el énfasis puesto en la ampliación de la cobertura educativa comienza una apertura - al

\footnotetext{
"Instituto de Filosofía y Estudios Educacionales. Facultad de Filosofía y Humanidades. Universidad Austral de Chile. Valdivia, Región de los Ríos, Chile. E-mail: alberto.moreno@uach.cl "Facultad de Ciencias de la Educación. Universidad de Granada. Granada, Granada, España. E-mail: ctriguer@ugr.es

"*Facultad de Ciencias de la Educación. Universidad de Granada. Granada, Granada, España. E-mail: erivera@ugr.es
} 
menos de forma discursiva - respecto a la preocupación por la calidad y equidad como focos centrales, que contrariamente a lo expuesto pone el énfasis en los resultados académicos obtenidos en pruebas estandarizadas. En EF queda reflejado en la reciente implementación del Sistema de Medición de la Calidad de la Educación (SIMCE) específico del área, que pretende evaluar la calidad a partir de la medición de los niveles de condición física y del índice de masa corporal en los estudiantes. En este contexto, la transformación de las desigualdades sociales y educativas tiene difícil encaje como una de las funciones de la EF, especialmente si la formación inicial de los profesores se mantiene anclada y sustentada en una visión tradicional del área, basada en estrategias reproductoras de un saber objetivo, observable y medible que den sentido al SIMCE.

Cuando hablamos de las funciones de la EF como asignatura del currículum escolar, podemos ver diferentes posturas y clasificaciones (MORENO 2011; MARTÍN 2009; DEVÍS; MOLINA 2004; DÍAZ 1994), que abren paso a lo que hemos venido entendiendo como "corrientes de la EF". Apoyándonos en los trabajos de Devís y Molina (DEVÍS; MOLINA 1998a; 1998b; 2004; DEVÍS 1994), tres van a ser las corrientes que permitieron dar sentido a la EF y a sus funciones durante el proceso de la modernidad. La primera entendía que la EF era 'educación de lo físico' y, por tanto, a partir de ella surgían los "[...] cuidados, usos y disciplina corporales, la regeneración física y mejora de la raza, la obediencia y la preocupación por la higiene y la salud corporal para hacer ciudadanos productivos y útiles a los estados modernos" (DEVÍS; MOLINA, 2004 , p. 2). En esta conceptualización podemos ver la importancia otorgada a la dimensión corporal del ser humano y la explícita separación entre la mente y el cuerpo, postura epistemológica aún presente en muchas de las propuestas actuales del área (TORO, 2007).

Esta dualidad se mantuvo, incluso, en la segunda de las conceptualizaciones. A pesar del esfuerzo por valorar la disciplina a partir de la consideración de que toda actividad motriz, por simple que fuera, llevaba implícita la disyunción entre pensamiento y voluntad 
para realizarla. Fue así como surge la conceptualización 'educar a través de lo físico'. Esta conceptualización amplió la mirada, intentando que la EF fuera algo más allá de lo puramente corporal, pero "[...] seguía orientada a funciones de tipo social de carácter extrínseco y conectadas a los valores dominantes de las sociedades capitalistas" (DEVÍS; MOLINA, 2004, p.38).

Fue Arnold (1991) quien propuso una nueva conceptualización que permitiera revalorizarla por ella misma y no por lo que se podría conseguir a través de ella. Cuando en una actividad importa tanto su realización como la manera de practicarla, se está entendiendo que fines y medios no están separados sino que ambos son intrínsecamente valiosos. En este punto es posible comprender la afirmación de Arnold (1991, p.23) al decir que: "[...] tanto la materia como el método se convierten en fines y que el método no se le considera simplemente como un medio de promover la materia". Su propuesta está fundada en tres dimensiones que delimitarían tres funciones propias de la EF escolar: 'educación sobre el movimiento', 'educación a través del movimiento' y 'educación en movimiento'. La primera se refiere a los conocimientos teóricos relativos al campo de la EF. La segunda, de carácter instrumental (extrínseco), centrada en los aspectos que podríamos desarrollar a partir del movimiento, asociados a él, y la última tiene un carácter totalmente propio, referida al hecho de los valores propios de la EF, como el autoconocimiento de quien la practica (ARNOLD 1991). Aún considerándolo como un aporte importante para revalorizar la EF, ésta sigue estando sustentada en una visión epistemológica dualista, y que se "[...] se elabora dentro de un vacío sociocultural que no entra a valorar factores y condicionantes sociales y contextuales que rodean a la educación física [...]"; por tanto podría terminar "reproduciendo el status quo imperante" (DEVÍS; MOLINA, 2004, p.39).

El tránsito de la modernidad a la postmodernidad ha originado que a la escuela, como institución social, le sea exigida una serie de funciones a cumplir. Según Devís y Molina (2004), estos cambios provocan que el currículo, creado para la reproducción material y cultural en la modernidad, se oriente hacia una escuela comprometida 
con procesos de transformación basados en la justicia social y en la reconstrucción del conocimiento. Siguiendo, nuevamente, a Devís y Molina (2004), podemos sintetizar las funciones de la EF, en la época postmoderna, en dos ideas claves: Justicia social y reconstrucción de los conocimientos y las experiencias. Respecto a la primera, la escuela tiene como uno de sus objetivos prioritarios la 'compensación de las desigualdades' (PÉREZ, 1993). En EF se reflejaría en orientar las acciones hacia la promoción de valores y actitudes tales como el respeto a las personas y la igualdad, facilitando que la EF sea capaz de favorecer prácticas positivas y beneficiosas de actividad física para los más necesitados. En referencia a la 'Reconstrucción de los conocimientos y las experiencias' que el alumnado tiene en su vida paralela y anterior a la escuela (PÉREZ 1993; 1998). "En el ámbito de la EF, la reconstrucción se refiere al conocimiento teórico y al conocimiento práctico o experiencial..." (DEVÍS; MOLINA, 2004, p.42). No olvidemos que desde hace algunos años, la compensación de las desigualdades de origen y la reconstrucción del conocimiento, figuran como dos áreas centrales para que profesores y profesoras de EF teoricen y construyan propuestas prácticas en los diferentes niveles del sistema educativo. En este sentido la pedagogía crítica, particularmente en el área de la EF, ha centrado sus esfuerzos en dichas líneas de acción (LÓPEZ; PÉREZ; MONJAS 2007; FRAILE 2004; LÓPEZ; MONJAS; PÉREZ 2003).

\section{Objetivos}

El trabajo presentado es producto de una investigación contextualizada en la educación superior chilena y realizada con docentes universitarios del área de EF. El objeto de la investigación es analizar sus percepciones, en relación a las funciones de la EF como asignatura curricular. Hablar de percepciones es aludir a "la configuración que el observador hace de objetos preceptúales mediante la distinción de clivajes operacionales en la conducta del organismo, al descubrir las interacciones de éste en el fluir de su correspondencia estructural con el medio" (MATURANA, 2004, p.65). El objeto de la investigación nos lleva a preguntarnos cómo 
los docentes universitarios del área de EF están pensando, imaginando, sintiendo y soñando las funciones de la asignatura y cómo están interpretando el quehacer educativo en el área. Este análisis, nos ayudará a comprender el fenómeno y mejorar la práctica educativa, tanto en la educación superior como en el resto niveles.

\section{Metodología}

Es un estudio fenomenológico interpretativo, realizado con metodología cualitativa. Esta elección se basa en el objeto de estudio y las preguntas que han guiado el proceso con la intención de identificar, comprender cualidades y características, lejos de una mirada cuantitativa. Para su descripción, interpretación y comprensión se han utilizado conceptos y relaciones entre ellos (STRAUSS; CORBIN, 2002). A su vez, el trabajo con grupos focales y la realización de entrevistas en profundidad, como técnicas de recolección de datos, posibilitaron analizar la información.

La selección de los participantes para los grupos focales (10 participantes en el primero y 9 en el segundo) se realizó a partir de criterios que permitieron representatividad en función de: (i) Profesores de diferentes zonas del país. (ii) Docentes universitarios que hayan tenido experiencias pedagógicas en otros niveles del sistema educativo. (iii) Hombres y mujeres. (iv) Profesores del practicum. Las entrevistas en profundidad se realizaron a 9 profesores universitarios de EF de diferentes universidades del país y la selección se realizó a partir de la estrategia de 'bola de nieve' . Se partió de una consulta a expertos en el área, que aportaron criterios previos para la selección, evitando el uso del azar. El principal criterio se orientó a seleccionar docentes universitarios de EF que estuvieran pensando, haciendo y/o publicando acerca de una EF diferente a la tecnocrática.

Para caracterizar a los participantes utilizamos los atributos: área donde realizan docencia; edad; experiencia docente; formación de postgrado; participación en proyectos de investigación y/o innovación educativa; sexo; tipo de institución ; universidad donde 
trabajan; zona geográfica. Esto nos permitió realizar cruces de información con el NVivo. Los procedimientos de análisis empleados se ajustaron a las propuestas de fragmentación y articulación de la "Grounded Theory" (VALLES 2007; STRAUSS; CORBIN 2002; GLASER; STRAUSS 1967). La estrategia ha sido en espirales de profundización, retrocediendo y avanzando de forma continua a lo largo del proceso. La primera tarea de nuestro análisis consistió en reducir los datos desde la simplificación, resumen y selección de la información.

Como señalan Strauss y Corbin (2002:110), en la primera codificación de los datos, "éste sería el proceso analítico por medio del cual se identifican los conceptos y se descubren en los datos sus propiedades y dimensiones". En la primera fase se realizó una lectura analítica de los datos seleccionado las unidades de significado previas en base a "criterios temáticos" (RODRÍGUEZ; GIL; GARCÍA, 1999, p. 207). El proceso seguido ha sido fundamentalmente inductivo (MILES; HUBERMAN 1994), aunque reconocemos cierta contaminación desde los guiones de los grupos focales y entrevistas.

En la segunda fase, se procedió a la síntesis y agrupamiento, procedimiento que permitió juntar varias unidades y códigos bajo un mismo tópico o concepto teórico (RODRÍGUEZ; GIL; GARCÍA 1999). Esta labor de síntesis estuvo orientada a una recreación de lenguaje, que corresponde en la literatura a la transformación de los datos (RUIZ 2003; STRAUSS; CORBIN 2002; RODRÍGUEZ; GIL; GARCÍA 1999; MARTÍNEZ 1999). Existiendo varios procedimientos, hemos optado por las representaciones gráficas (figura 1) o imágenes visuales de la relación entre conceptos (STRAUSS; CORBIN 2002). La tercera fase se materializa en la obtención de resultados y conclusiones, que consistió fundamentalmente en la contextualización y constatación de los hallazgos alcanzados con otros estudios, para finalmente elaborar un informe narrativo.

La emergencia de las categorías, desde una primera codificación (creación de nodos libres) hasta llegar a la creación de un árbol de categorías (nodos ramificados), se manifiesta en la indexación 
inductiva (categorización) desde la lectura de la transcripción de los grupos focales. La construcción de los primeros "nodos libres" sirvieron de primer borrador para elaborar el árbol de nodos ramificados alcanzada por esta vía.

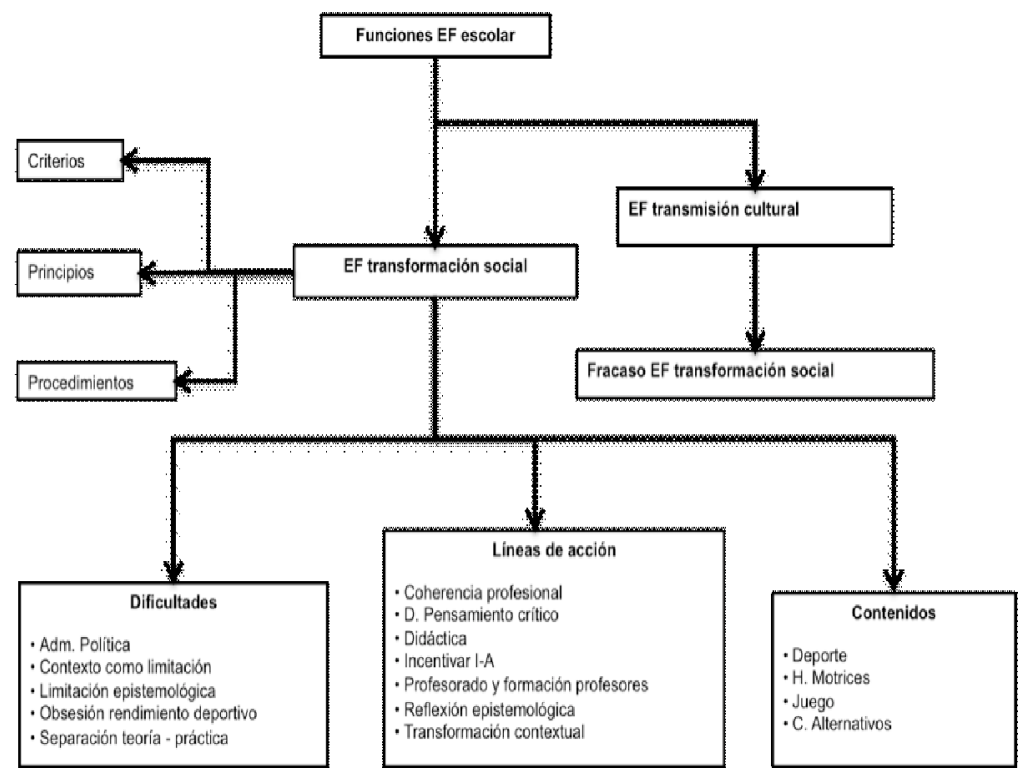

Figura 1. Mapa conceptual - Árbol de categorías

\section{AnÁlisis y Resultados}

\subsection{Primer ACercamiento}

La aproximación cuantitativa que realizamos inicialmente se justifica como la puerta de entrada que nos permitirá abordar el análisis cualitativo desde el respeto a los participantes. El nivel de participación, en ambos grupos focales, está mediatizado por la concentración del discurso por un determinado número de participantes. Respecto al peso de las temáticas presentes (figura2), 
se advierte que la "EF como transformación social" y "Dificultades de la EF para la transformación social" acapararon más del 50\% del discurso global.

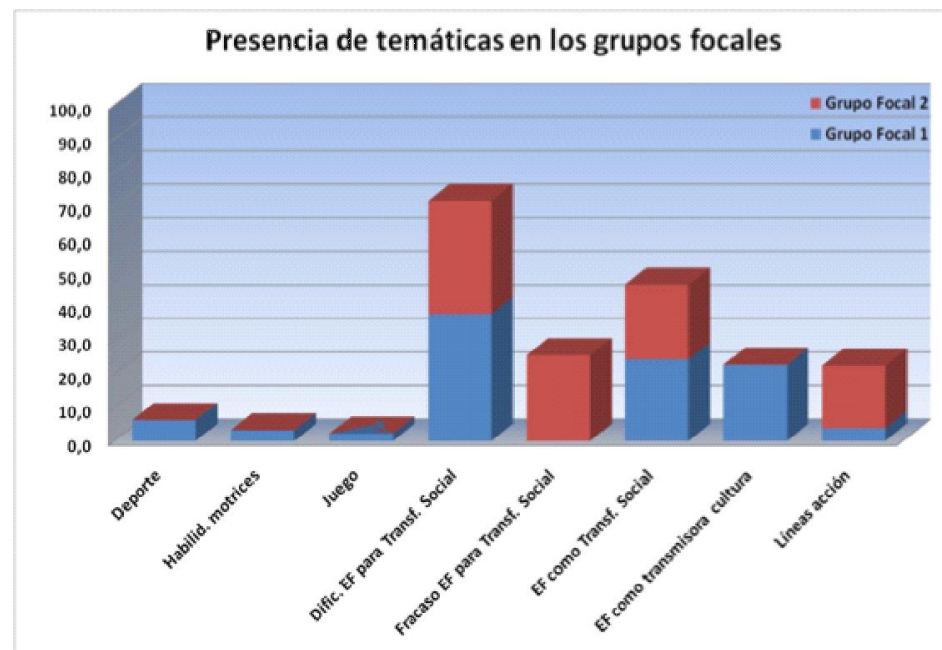

Figura 2: Presencia de temáticas en los grupos focales

Puede afirmarse que los participantes construyen un discurso que gira en torno a la idea que la EF tiene dos funciones principales: La necesidad de entender la EF como una asignatura obligada a cooperar en la transmisión de la cultura existente y ayudar en la transformación los aspectos culturales que pudieran estar en entredicho. Pero advierten que esta segunda función está muy lejos de ser alcanzada. Los participantes reconocen en la EF tres contenidos relevantes a partir de los cuales trabajar estas dos funciones: "deporte", "habilidades motrices" y "juego".

Los sujetos entrevistados (figura3), por su parte, no sólo vislumbran una EF como agente de transformación social, sino que además identifican los procedimientos, principios y criterios importantes para alcanzarla. 


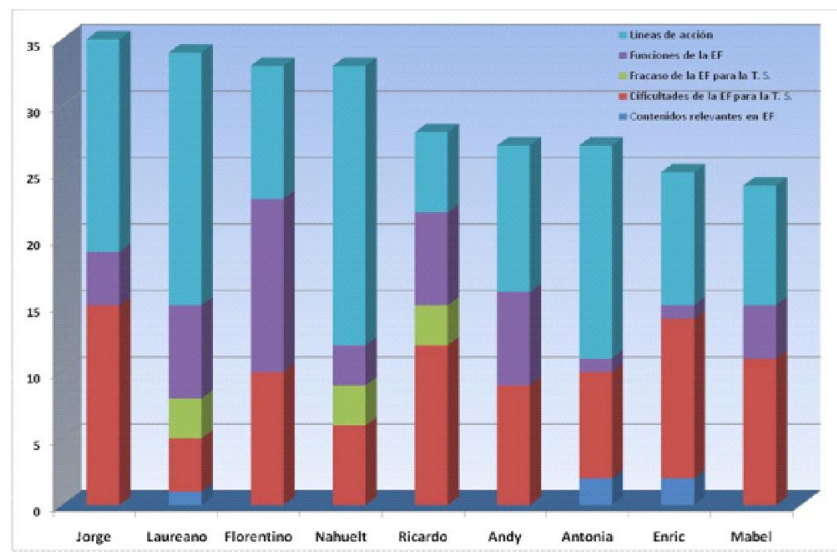

Figura 3: Temáticas abordadas por participantes entrevistados

Para hacer posible lo comentado hasta el momento se requieren profesores de EF que consideren "cambiar la lógica" a partir de la cual desarrollar su labor profesional, considerando "relaciones distintas (...), espacios distintos (...), formas de ver el mundo distintas". Para que este cambio de lógica se haga real es relevante "sentarse a consensuar (...) no votar (...), porque la votación termina como anulando al que perdió, al que tuvo menos voto, y el consenso es bueno, ..." (Florentino).

Las dificultades enunciadas por ellos son variadas, pero hay una que llama poderosamente la atención, pues representa un elevado porcentaje $(45 \%)$ en relación a las otras: la "Limitación Epistemológica".

Cuando hacen referencia a las líneas de acción a seguir, mencionan la didáctica como mecanismo constructor de una realidad determinada, y sería éste el principal factor a tener en cuenta para conseguir una EF que ayude en la transformación social. "Asumes, no como el que a va a decir lo que hacen (...) No puedes ver la didáctica como algo que está afuera, tienes que ver la didáctica como algo que emerge de ti, como sujeto". A estos efectos, resulta indispensable que los docentes entiendan que el quehacer docente 
no se limita a enseñar contenidos disciplinares, sino que organizan, definen, proyectan, sueñan y construyen realidades, es decir, "gestionan (...), [son los que hacen] el vínculo entre un contenido, un implemento, un espacio (...)" (Nahuelt).

La intencionalidad pedagógica se centra en el desarrollo de un tipo de pensamiento que sea capaz de "enfrentar el sistema y producir un cambio", a partir del cual no se evalúe negativamente la realidad, sino como una "estrategia" que permita generar "un pensamiento crítico propositivo", propositivo: qué es lo que se "quiere cambiar" y coadyuvante a generar "consensos" (Laureano).

\begin{tabular}{|c|c|c|c|c|c|c|c|c|c|c|c|c|c|c|c|}
\hline & \multicolumn{2}{|c|}{ Área } & \multicolumn{2}{|c|}{$\begin{array}{l}\text { Exp. } \\
\text { Básica }\end{array}$} & \multicolumn{3}{|c|}{ Exp. Media } & \multicolumn{3}{|c|}{ Exp. Universidad } & \multicolumn{3}{|c|}{ Formación } & \multicolumn{2}{|l|}{$\begin{array}{l}\mathrm{I} \\
\mathrm{n} \\
\mathrm{s} \\
\mathrm{t} \\
\mathrm{i} \\
\mathrm{t} \\
\mathrm{u} \\
\mathrm{c} \\
\mathrm{i} \\
\mathbf{o} \\
\mathrm{n}\end{array}$} \\
\hline & $\begin{array}{l}\mathbf{P} \\
\mathrm{e} \\
\mathbf{d} \\
\mathrm{a} \\
\mathrm{g} \\
\mathbf{o} \\
\mathrm{g} \\
\mathrm{i} \\
\mathrm{c} \\
\mathrm{a}\end{array}$ & $\begin{array}{l}\text { E } \\
\text { s } \\
\text { p } \\
\text { e } \\
\text { c } \\
\text { i } \\
\text { a } \\
\text { } \\
\text { i } \\
\text { d } \\
\text { a } \\
\text { d }\end{array}$ & $\begin{array}{l}\mathbf{H} \\
\mathbf{a} \\
\mathbf{s} \\
\mathbf{t} \\
\mathbf{a} \\
\mathbf{5} \\
\\
\mathbf{a} \\
\tilde{\mathbf{n}} \\
\mathbf{o} \\
\mathrm{s}\end{array}$ & $\begin{array}{l}\mathbf{5} \\
\mathbf{a} \\
\mathbf{1} \\
\mathbf{0} \\
\\
\mathbf{a} \\
\tilde{\mathbf{n}} \\
\mathbf{0} \\
\mathrm{s}\end{array}$ & $\begin{array}{l}\text { H } \\
\mathbf{a} \\
\mathbf{s} \\
\mathbf{t} \\
\mathrm{a} \\
\\
5 \\
\\
\mathbf{a} \\
\tilde{\mathbf{n}} \\
\mathbf{0} \\
\mathrm{s}\end{array}$ & $\begin{array}{l}\text { D } \\
\text { e } \\
\mathbf{5} \\
\mathbf{a} \\
\mathbf{1} \\
\mathbf{0} \\
\mathbf{a} \\
\tilde{\mathbf{n}} \\
\mathbf{o} \\
\mathrm{s}\end{array}$ & $\begin{array}{l}\mathbf{1} \\
\mathbf{5} \\
\mathbf{a} \\
\mathbf{2} \\
\mathbf{0} \\
\mathbf{a} \\
\tilde{\mathbf{n}} \\
\mathbf{o} \\
\mathrm{s}\end{array}$ & $\begin{array}{l}5 \\
\text { a } \\
1 \\
0 \\
\\
\mathbf{a} \\
\tilde{\mathbf{n}} \\
0 \\
\mathrm{~s}\end{array}$ & $\begin{array}{l}1 \\
0 \\
\text { a } \\
1 \\
5 \\
\\
\mathbf{a} \\
\tilde{\mathbf{n}} \\
\mathbf{0} \\
\mathrm{s}\end{array}$ & $\begin{array}{l}\text { M } \\
\mathbf{a} \\
\mathbf{s} \\
\mathbf{d} \\
\mathbf{e} \\
\mathbf{2} \\
\mathbf{0} \\
\mathbf{a} \\
\tilde{\mathbf{n}} \\
\mathbf{0} \\
\mathbf{s}\end{array}$ & $\begin{array}{l}\text { M } \\
\text { a } \\
\text { g } \\
\mathbf{i} \\
\mathbf{s} \\
\text { t } \\
\text { e } \\
\text { r }\end{array}$ & $\begin{array}{l}\text { D } \\
\text { o } \\
\text { c } \\
\text { t } \\
\text { o } \\
\text { r }\end{array}$ & $\begin{array}{l}\text { L } \\
\text { i } \\
\text { c } \\
\text { e } \\
\text { n } \\
\text { c } \\
\text { i } \\
\text { a } \\
\text { t } \\
\text { u } \\
\text { r } \\
\text { a }\end{array}$ & $\begin{array}{l}\text { Con } \\
\text { sejo } \\
\text { Rec } \\
\text { t. }\end{array}$ & $\begin{array}{l}\text { N } \\
\mathbf{o} \\
\\
\mathrm{C} \\
\mathbf{0} \\
\mathrm{n} \\
\mathrm{s} \\
\mathrm{e} \\
\mathrm{j} \\
\mathbf{0} \\
\mathrm{R} \\
\mathrm{e} \\
\mathrm{c} \\
\mathrm{t}\end{array}$ \\
\hline Líneas de acción & $\begin{array}{c}5,7 \\
6\end{array}$ & $\begin{array}{c}6,2 \\
2\end{array}$ & 8,9 & $\begin{array}{c}5,0 \\
6\end{array}$ & $\begin{array}{c}6,9 \\
8\end{array}$ & $\begin{array}{c}4,9 \\
7\end{array}$ & 11 & $\begin{array}{c}8,3 \\
8\end{array}$ & $\begin{array}{c}6,8 \\
1\end{array}$ & $\begin{array}{c}4,6 \\
1\end{array}$ & $\begin{array}{c}6,9 \\
6\end{array}$ & $\begin{array}{c}4,1 \\
9\end{array}$ & $\begin{array}{c}4,7 \\
1\end{array}$ & 11,7 & 3,81 \\
\hline $\begin{array}{l}\text { Dificultades de } \\
\text { la EF para la TS }\end{array}$ & $\begin{array}{c}6,5 \\
7\end{array}$ & $\begin{array}{c}6,3 \\
3\end{array}$ & $\begin{array}{c}4,6 \\
2\end{array}$ & $\begin{array}{c}6,9 \\
3\end{array}$ & 5,6 & $\begin{array}{c}7,0 \\
6\end{array}$ & $\begin{array}{c}4,3 \\
8\end{array}$ & 5,6 & $\begin{array}{c}9,1 \\
2\end{array}$ & $\begin{array}{c}5,6 \\
9\end{array}$ & $\begin{array}{c}6,3 \\
6\end{array}$ & $\begin{array}{c}5,4 \\
7\end{array}$ & $\begin{array}{c}8,0 \\
3\end{array}$ & 16,5 & 1,98 \\
\hline $\begin{array}{l}\text { Funciones de la } \\
\text { EF }\end{array}$ & $\begin{array}{c}9,1 \\
9\end{array}$ & $\begin{array}{c}5,8 \\
3\end{array}$ & $\begin{array}{c}7,4 \\
4\end{array}$ & $\begin{array}{c}5,6 \\
9\end{array}$ & $\begin{array}{c}11, \\
8\end{array}$ & $\begin{array}{c}3,7 \\
2\end{array}$ & $\begin{array}{c}3,9 \\
4\end{array}$ & $\begin{array}{c}4,8 \\
1\end{array}$ & $\begin{array}{c}11 \\
2\end{array}$ & $\begin{array}{c}4,9 \\
9\end{array}$ & $\begin{array}{c}7,3 \\
1\end{array}$ & $\begin{array}{c}2,6 \\
2\end{array}$ & $\begin{array}{c}5,2 \\
5\end{array}$ & 13,1 & 3,19 \\
\hline $\begin{array}{l}\text { Fracaso de la } \\
\text { EF para la TS }\end{array}$ & 0 & $\begin{array}{c}7,5 \\
2\end{array}$ & 15 & $\begin{array}{c}3,7 \\
6\end{array}$ & $\begin{array}{c}7,5 \\
2\end{array}$ & $\begin{array}{c}3,7 \\
6\end{array}$ & $\begin{array}{c}22, \\
6\end{array}$ & $\begin{array}{c}7,5 \\
2\end{array}$ & 0 & $\begin{array}{c}9,0 \\
2\end{array}$ & $\begin{array}{c}9,6 \\
7\end{array}$ & 0 & 0 & 7,52 & 6,44 \\
\hline $\begin{array}{l}\text { Contenidos } \\
\text { relevantes EF }\end{array}$ & 0 & $\begin{array}{c}9,2 \\
4\end{array}$ & $\begin{array}{c}5,5 \\
5\end{array}$ & $\begin{array}{c}11, \\
1\end{array}$ & $\begin{array}{c}5,5 \\
5\end{array}$ & 11 & 0 & 11 & 0 & $\begin{array}{c}9,9 \\
8\end{array}$ & $\begin{array}{l}11 \\
9\end{array}$ & 0 & 0 & 22,2 & 2,38 \\
\hline $\begin{array}{l}\text { Nivel } \\
\text { porcentual de } \\
\text { Participación }\end{array}$ & 4,3 & $\begin{array}{c}7,0 \\
3\end{array}$ & $\begin{array}{c}8,3 \\
1\end{array}$ & $\begin{array}{c}6,5 \\
1\end{array}$ & $\begin{array}{c}7,4 \\
9\end{array}$ & $\begin{array}{c}6,1 \\
2\end{array}$ & $\begin{array}{c}8,3 \\
7\end{array}$ & $\begin{array}{c}7,4 \\
8\end{array}$ & $\begin{array}{c}5,4 \\
2\end{array}$ & $\begin{array}{c}6,8 \\
6\end{array}$ & $\begin{array}{c}8,4 \\
4\end{array}$ & $\begin{array}{c}2,4 \\
6\end{array}$ & 3,6 & 14,2 & 3,56 \\
\hline
\end{tabular}

Tabla 1: Matriz de atributos con valores ponderados y expresión de porcentajes 
Entrando en el análisis del cruce entre los atributos de los participantes y las grandes temáticas presentes en el discurso (tabla 1), nos detendremos en aquellos más significativos para el artículo.

La mayor experiencia en docencia universitaria no implica percibir las funciones de la EF orientada a la transformación y mejora social. Se entiende que habría varios motivos, hipótesis emergentes, que podrían ayudar a explicar esta situación. Los docentes con más años de experiencia en el contexto universitario son los que, casualmente, poseen menos experiencia en los otros niveles del sistema educativo y, por tanto, los que conocen el resto de niveles de forma más tangencial. Esto nos lleva a pensar en la dificultad de llevar a la práctica aquello que estos docentes están pensando y percibiendo de la EF. Se hace patente algo a lo que los participantes suelen aludir como dificultad de la EF para contribuir, desde el área, a la transformación social: la separación entre teoría y práctica. Esta situación también aparece relacionada con lo propuesto por gran parte de los entrevistados: el paradigma dicotómico, de origen cartesiano, que aún persiste en la disciplina.

La relación entre universidades pertenecientes, o no, al Consejo de Rectores es algo que se ha visualizado en bastantes cruces. Puede percatarse de que las instituciones que sí forman parte de este conglomerado, son aquellas que reciben la mayor parte de los fondos del Estado, focalizando sus tareas en las áreas de docencia, investigación y extensión, a diferencia del resto, cuyo mayor foco está puesto en la docencia. A la vez, las primeras poseen un gran número de académicos a jornada completa, en las segundas abundan los profesores contratados por horas, por lo que trabajan en varias instituciones para completar jornada (Organización para la Cooperación y el Desarrollo Económicos 2009). Los docentes de universidades pertenecientes al Consejo de Rectores, pueden dedicar parte de su jornada a labores de perfeccionamiento e investigación. Esta situación podría explicar que los otros profesores estén menos interiorizados de las líneas de acción a seguir para hacer, de la EF, una asignatura que tienda al trabajo por la transformación y mejora social. 
Centrándonos en la funciones de la EF, como nodo principal a partir del cual establecer ciertas diferencias entre los participantes, señalar que los que poseen una experiencia docente universitaria entre diez y quince años son lo que hacen mayor alusión a las mismas $(11,2 \%)$, en detrimento de los que tienen una experiencia entre cinco y diez años $(4,8 \%)$ y aquellos con más de veinte años (5\%).

En relación a las dificultades existentes a nivel social, escolar y de la EF para ayudar, colaborar y orientar la práctica pedagógica hacia la transformación y mejora social, los docentes universitarios del Consejo de Rectores referencian dicha situación muchísimas más veces $(16,5 \%)$ que los no pertenecientes $(2 \%)$. Esto muestra que la pertenencia al Consejo de Rectores pareciera ser una 'variable' que influye en una mirada más social y transformativa de la educación, fruto de las facilidades para la investigación y el estudio, lo que permitiría un mejor reconocimiento de las dificultades con las que nos encontramos los docentes en la puesta en práctica de nuestras concepciones y visiones educativas. Se produce una clara identificación del fracaso de la EF frente a la función de transformación social que debiera ejercer. Datos que los podemos vislumbrar con mayor o menor intensidad en base a la experiencia del docente.

\subsection{SEGUNDO ACERCAMIENTO: RECUPERANDO LA VOZ DE LOS PARTICIPAN- TES}

Analizada la foto aérea desde la aproximación cuantitativa, vamos a profundizar en tres aspectos relevantes para los participantes en la investigación y que nos revelan sus percepciones en relación a las funciones de la EF como asignatura del curriculum escolar:

"Funciones de la EF

"Dificultades profesionales para la transformación social desde el área

"Importancia de la reflexión como mecanismo generador de cambios. 


\subsubsection{FUNCIONES DE LA EDUCACIÓN FÍSICA COMO ASIGNATURA DEL CURRÍCULUM ESCOLAR}

El primer foco abierto por los participantes respecto a las funciones de la EF es la necesidad de crear hábitos saludables de práctica de actividad física. Para Manu, la problemática del "ausentismo laboral" es debido a la inexistencia de una "condición física adecuada". Para él, la ausencia de un "hábito desarrollado tempranamente" agudiza la problemática "porque estas cuestiones de hábitos si no se inculcan a los 10, 11, 12 ó 13 años...". Manu nos propone "trabajar con el cuerpo de los niños" y así conseguiremos que "tengan un confort, un bienestar, una mejor calidad de vida". Entiende que la calidad de vida tiene que ver, casi exclusivamente, con una perspectiva biomédica, sin atender a toda la complejidad del ser humano en su totalidad e integridad. Rafael establece una relación directa entre "el desarrollo de [las] capacidades físicas y (...) todos los elementos de salud". Y Antonio sugiere trabajar, en la formación de profesores, la relación entre actividad física y salud, para lo cual se refiere, casi exclusivamente, a "los problemas de obesidad infantil".

En este acercamiento al concepto de salud reproduciendo lo que se entiende socialmente por ella, Miguel señala que se podría "utilizar la actividad física, la actividad motriz, o como queramos llamarle...", es decir, que la EF se convertiría en "un medio para desarrollar esos valores" y no un fin en sí misma. Se rescata la idea de una EF para educar "a través de los físico" sin realzar el valor intrínseco propio del área (DEVÍS; MOLINA, 1998a; 2004; ARNOLD 1991).

Ramón abre un segundo foco de atención: la transmisión de valores a través de la EF. "Vamos a ocupar un papel importante desde el punto de vista de que los estudiantes se vayan comprometiendo con los valores que están siendo transmitidos a través de la actividad física". Manu identifica una diferencia sustancial entre la EF y las otras asignaturas del curriculum escolar pues, en nuestra área, "los valores se viven, no se escuchan, no se repiten sin sentido, se viven". Es importante que los estudiantes se muevan, estén activos, pues "algunos profesores tienen a los niños 
parados hablándoles y conversándoles de estos conceptos". No perdamos tiempo en conversar sobre los valores que se están viviendo, ya que la EF "es una cosa activa" y no debemos "parar una clase, detener a los niños... tenerlos sentados media hora hablando de la importancia de los valores". La solución: vivirlos y no conversarlos, teniendo en cuenta que los valores a desarrollar es diferente en "un estrato social $\mathrm{ABC} 1$ que en un estrato social más vulnerable, hay intereses y necesidades distintas" (Manu).

Finalmente, en los discursos de los participantes podemos ver un tercer foco de atención: atender a la transformación de aquellos aspectos sociales que impiden un verdadero desarrollo humano. En este sentido los discursos son dispares; mientras Manu, focalizando la atención en el "problema del bulling, de la delincuencia, de la tendencia a la drogadicción en los escolares, en sectores vulnerables especialmente hablando". Fabiola plantea que "hay que cambiar..., nuestro objetivo principal no es que corran más rápido o que metan gol", lo realmente importante es la "formación a través de la pelota". Para Ramón lo importante es generar desde la EF una "buena convivencia", para él olvidada:

[...] el rol formativo que cumple la EF tiene que ver con buscar fórmulas para que el niño no solamente desarrolle competencias en el hacer, que es una cosa a la que nosotros, quizás, durante mucho tiempo nos dedicamos, sino con el saber, el saber convivir, que son elementos fundamentales.

¿Cómo abordar la función de transformación social desde la EF? No es tarea fácil. Algunos participantes nos aportan ideas que pudieran ayudar en dicho trabajo. José plantea el camino: ser "capaz de enseñarle a niños y jóvenes a hacer actividad física sistemática permanentemente y de forma voluntaria..." Breno señala que los contenidos a aprender son importantes, pero no lo central; lo básico para contribuir a la transformación social estaría en el "desarrollo afectivo", pues ello "transcenderá sobre las actitudes a desarrollarse y que puedan propender a la transformación social". José señala un tercer aspecto, el de la "diversidad, que es la aceptación de todo, que no todos somos iguales, somos diversos, somos... somos seres 
únicos", independientemente de las características físicas, psicológicas, sociales, económicas, históricas, etc. Arturo ahondando en el tema cree que es central en la EF y es ahí "donde encontramos una primera discusión en relación al tema del alto rendimiento deportivo". Una cuarta característica que ayudaría en la función de transformación social de la EF sería, según Manu, el trabajo colaborativo con profesionales de otras áreas, pues "no podemos olvidar que estamos rodeados de otros colegas que también están intentando hacer algo parecido desde el punto de vista de inculcar valores...".

\subsubsection{DiFICULTADES PARA LA TRANSFORMACIÓN SOCIAL}

¿Qué dificultades encuentran los participantes? Veamos las principales:

Lógica social. Para Javier la situación social actual, donde los intereses económicos prevalecen sobre otros, nos impide darle una verdadera solución a las problemáticas sociales, "la sociedad está enferma" y eso hace que nosotros no seamos la solución, sino "parte de ella... hay todo un tema cultural por cambiar".

Administración política. Miguel comenta que "este verano se hacen dos gimnasios más y, por una cuestión política, sólo uno de los gimnasios se puede utilizar, pues no los facilitan para la comunidad. Motivos específicos no los tengo.". Esto, unido a la misma organización administrativa, y a que no hay equipamientos suficientes dificulta un mejor cumplimiento de las funciones propias del área,

$$
\begin{aligned}
& \text { [...] incluso [...] tenía como misión [...] generar } \\
& \text { en las tardes, como Jornada Escolar Completa, algún } \\
& \text { programa de actividades... Resulta que las } \\
& \text { actividades se convirtieron en reforzar las actividad } \\
& \text { de la mañana... entonces ahí también tenemos un } \\
& \text { problema dentro de la misma organización } \\
& \text { administrativa, cómo se entregan las materias y } \\
& \text { cómo se maneja esto. (Manu). }
\end{aligned}
$$

Modelo de profesor. Para Valentín, es "el profesional de la EF escolar y el modelo que representa para los estudiantes".Carmen 
argumenta que "la poca innovación pedagógica existente en EF" dificulta adecuar las clases a los verdaderos intereses de los alumnos y alumnas. Manu conviene en afirmar que la casi"inexistencia de profesionales de la EF escolar en la Enseñanza General Básica" es el principal impedimento desde el criterio del "profesorado como barrera".

Obsesión por el rendimiento deportivo. Fabiola señala la última barrera a la hora de querer plantear una EF escolar sustentada en la idea de generar cierta transformación social: "ansiedad por alcanzar el rendimiento deportivo", lo que hace que los profesores se sientan presionados por, sobre todo, ganar campeonatos, provocando que desatiendan su mirada de otros factores más relevantes. Dicha obsesión está afectando al alumnado en su placer intrínseco por el juego. Una muestra de ello -dice Fabiola- es que en "tercero medio encontramos grupos de 30 niños de los que 25 declaran estar enfermos para no hacer EF".

\subsubsection{AlgunAS ORIENTACIONES PARA LA TRANSFORMACIÓN SOCIAL DESDE LA EF ESCOLAR}

Existe una angustiosa realidad para los profesores noveles de EF cuando ingresan al sistema escolar; se produce una fuerte contradicción entre los intereses por "generar un cambio" de carácter cualitativo y las lógicas que siguen los docentes experimentados. La caracterización del cambio tiene como principio fundamental el instaurar la reflexión como mecanismo orientador que invite, incentive y se responsabilice por la amplitud de ella, que debiera abordar diferentes dimensiones: desde "la reflexión del aula, la disciplina", hasta dimensiones más amplias y contextuales como sus "componentes ontológicos, epistemológicos", a la vez que aspectos concretos como "los metodológicos".

Jorge invita a una reflexión epistemológica a partir de la cual entendamos que cuerpo y mente no son dos realidades diferenciadas, pues ello es uno de los estigmas de nuestra área de conocimientos. 
Mabel cree posible la "reconstrucción de significados y [el] cuestionamiento" por la inexistencia de experiencias previas y referentes de los estudiantes para docentes de EF; esto podría contribuir a la transformación y mejora social. Mabel sueña con los posibles caminos a seguir en el trabajo pedagógico con estudiantes universitarios, principalmente en los primeros años de formación, para lo cual se hace indispensable la consecuencia profesional y la minuciosa "articulación entre teoría y práctica". La actividad física transformadora comienza por la transformación de la persona, en toda su complejidad, y por el cuerpo del participante, pues desde él se posibilita aprender más de uno mismo y de su entorno. "dos cosas son fundamentales, (...) que la actividad física sea una actividad transformadora en la integridad de la persona, cómo desde mi cuerpo aprendo más de mí, cómo desde mi motricidad mejoro mi identidad y a partir de eso me conozco". El cuerpo se convierte para Mabel en el centro del trabajo pedagógico, pues según ella los seres humanos escribimos "el libro de nuestro cuerpo" y en él encontramos la experiencia humana vivida, "todo lo tenemos ahí, la violencia, la dulzura (...)".

Para operacionalizar estas experiencias en el quehacer pedagógico se hace necesario una "práctica más reflexionada", lo que sin duda requiere de "normas acordadas" entre los diferentes actores del quehacer educativo (Mabel).

\section{Discusión Y CONCLUSIONES}

Las funciones de la EF serían, principalmente, dos: la transmisión de la cultura existente en la sociedad contemporánea, y hacer un uso de la EF escolar para trabajar en aquellos aspectos de la misma sociedad que restringen y dificultan un desarrollo humano integral, complejo y completo. Ambas funciones aparecen, en el discurso de los participantes, como dos realidades complementarias, aunque también, en otras ocasiones, como realidades dicotómicas y contradictorias. 
La EF como transmisora de la cultura existente es consecuencia del dualismo cartesiano, aún presente en su sustento teórico. La disciplina es percibida como un área con una fundamentación epistemológica cuya intencionalidad pedagógica está orientada a adiestrar el cuerpo y dominarlo, resistiendo a modificarse a sí mismo $\mathrm{y}$, por tanto, resistiéndose a contribuir en la disminución de las desigualdades de origen (PEDRAZ 2007; TORO 2007) Su historia, como asignatura del currículum escolar, la ha posicionado en un paradigma que no le corresponde, ocasionando que sus contenidos e intencionalidades educativas giren en torno al desarrollo de hábitos considerados positivos por la sociedad, prescindiendo de una conceptualización crítica que lo fundamente.

Contrariamente a esta forma de entender y visualizar la EF, es posible encontrar una función de la asignatura más ligada al ámbito de la transformación social y educativa. Esta función, según nuestra investigación, está más ligada a un deber ser y a una aspiración todavía no lograda, pero en vías de desarrollo (MORENO 2011). Tal afirmación la hacemos desde el convencimiento que el trabajo pedagógico por la transformación social está ligado a una serie de aspectos susceptibles de ser intencionados desde la EF escolar.

Es preciso revisar el sustento epistemológico del área. La reflexión acerca de cómo estamos entendiendo el objeto de conocimiento de la EF, los conceptos de cuerpo y movimiento como apoyos de nuestro accionar pedagógico, es indispensable. Difícilmente podremos modificar nuestras prácticas si nuestra reflexión sigue los derroteros tradicionales del área (BARBERO 2007; KIRK 2004). Esto implica asumir un cambio en dos direcciones: nuevas reflexiones podrían ser el inicio de nuevas práctica, pero nuevas prácticas también podrían ser el origen de nuevas reflexiones. Su factibilidad aparece vinculada con acciones destinadas a revelar la coherencia profesional como un aspecto importante, en tanto que hacer y decir no pueden ser dos realidades independientes, sino que deben acompañarse congruentemente. 
O papel da Educação Física escolar no século xxi. um olhar desde o docente universitário.

Resumo: Neste trabalho são analisadas, qualitativamente, as percepções de 28 professores universitários da Faculdade de Educação Física em relação às funções da educação física, aprofundandose na função da transformação das desigualdades de origem. Em geral, os procedimentos de análises usados cingem-se às propostas da fragmentação e da articulação da "Grounded Theory". Conclui-se que a função da transformação social da educação física está relacionada com o dever de ser profissional, que ainda está concretizando-se, e estabelece-se uma série de princípios pedagógicos centrados na necessidade de revisar a sustentação epistemológica da mesma.

Palavras chave: Educação Física. Papel profissional. Responsabilidade Social.

The role of Physical Education in schools in 21st century. the university professor's view.

Abstract: In this paper we present a qualitative analysis of the perceptions of 28 university professors related to the functions of the Physical Education. We focus on the transformational function of the inequalities of origin. Generally, the procedures of the applied analysis adhered to the fragmentation and articulation proposed by the "Grounded Theory." We conclude that the social transformational function of the Physical Education is related to a sense of duty to become a professional not yet settled. We establish several pedagogical principles centered in the requirement to revise its epistemological support.

Keywords: Physical Education. Professional role. Social responsability 


\section{REFERENCIAS}

ARNOLD, Arnold. Meaning in movement, sport and physical education. London: Heinemann. 1991.

BARBERO, José. Capital(es) corporal(es) que configuran las corrientes y/o contenidos de la educación física escolar. Ágora para la Educación Física y el Deporte, Valladolid, n.4 y 5, p. 21-38, 2007.

DEVÍS, José. Educación física y desarrollo del currículum: un estudio de casos en investigación colaborativa. Tesis (doctoral), Departamento de Didáctica y Organización Escolar, Universitat de Valencia. Valencia, 1994.

DEVÍS, José; MOLINA, Joan. Funciones de la educación física en el currículum escolar. En: GARCÍA, A; RUIZ, F; CASIMIRO, A. La enseñanza de la Educación Física y el Deporte escolar. Málaga: I.A.D. Junta de Andalucía, 1998a. p. 48-51.

DEVÍS, José; MOLINA, Joan. Educación física escolar: funciones, racionalidad práctica e ideología. En: VILLAMÓN, Miguel. La Educación Física en el currículum de Primaria. Valencia: Generalitat Valenciana, 1998b. p. 13-32

DEVÍS, José; MOLINA, Joan. Las funciones de la educación física escolar: de la modernidad a la postmodernidad. En: CAPARROZ, A; ANDRADE, N. Investigação e intervenção. Vitória: LESEF/UFFS y NEPECC/UFU, 2004. p. 35-49.

DÍAZ, Jordi. El currículum de la Educación física en la reforma educativa. Barcelona: Inde, 1994.

FRAILE, Antonio. Didáctica de la Educación Física: una perspectiva crítica y transversal. Madrid: Biblioteca Nueva, 2004.

FREY, Lawrence R; BOTAN, Carl H; FRIEDMAN, Paul G; KREPS Gary L. Investigating Communication: an introduction to research methods. Needham Heights, Massachussets: Allyn \& Bacon, 2000.

GLASER, Barney; STRAUSS, Anselm. The discovery of grounded theory. Strategies for qualitative research. Chicago: Aldine, 1967.

KIRK, David. Towards a critical history of the body, identity and health: Corporal power and school practice. En: EVANS, Jhon; DAVIES, Brian; WRIGHT, Jan. (Coords.). Body knowledge and control: Studies in the sociology of physical education and health. Londres: Routledge, 2004. p. 52-67.

LÓPEZ, Víctor; MONJAS, Roberto; PÉREZ, Ángel. Buscando alternativas a la forma de entender y practicar la educación física escolar. Barcelona: Inde, 2003. 
LÓPEZ, Víctor; PÉREZ, Ángel; MONJAS, Roberto. La atención a la diversidad en el área de Educación Física. La integración del alumnado con necesidades educativas específicas, especialmente el alumnado inmigrante y de minorías étnicas. Revista Educación Física y Deportes, Buenos Aires, v.11, n.106, 2007. Disponible en: < http://www.efdeportes.com/efd106/la-atencion-a-la-diversidad-en-educacionfisica.htm>. Acceso en: 12 abr. 2010.

MARTíN, Francisco Jesús. Competencias Básicas y Funciones de la Educación Física en la LOE. Revista Digital Innovación y Experiencias Educativas, Madrid, n. 16, 2009. Disponible en: www.csi-csif.es/.../FRANCISCO \%20JESUS _MARTIN_1.pdf. Acceso en: 30 mar. 2010.

MARTÍNEZ, Miguel. La investigación cualitativa etnográfica en educación: Manual teórico - práctico. México: Trillas, 1999.

MATURANA, Humberto. Desde la biología a la psicología. Santiago de Chile: Universitaria, 2004.

MORENO, Alberto. Percepciones del profesorado universitario en relación a la función de transformación de la educación física como asignatura de curriculum escolar: el caso de Chile. Tesis (Doctoral). Granada: Universidad de Granada, 2011.

OCDE. Informe Revisión de Políticas Nacionales de Educación. La Educación Superior en Chile, 2009. Disponible en: http://feuc.cl/wp-content/uploads/2011/07/ Informe-OCDE-ES-en-Chile.pdf. Acceso en: 11 ago. 2010.

PEDRAZ, Vicente. El cuerpo sin escuela: proyecto de supresión de la educación física escolar y qué hacer con su detritus. Revista Ágora para la Educación Física y el Deporte, Valladolid, v. 4, 5, p. 57-90, 2007.

PÉREZ, Ángel. Las funciones sociales de la escuela: de la reproducción a la reconstrucción crítica del conocimiento y la experiencia. En: GIMENO, José; PÉREZ, Ángel. Comprender y transformar la enseñanza. Madrid: Morata, 1993. p. 17-33.

PÉREZ, Ángel. La cultura escolar en la sociedad neoliberal. Morata: Madrid, 1998.

RODRÍGUEZ, Gregorio; GIL, Javier; GARCÍA, Eduardo. Metodología de la investigación cualitativa. Málaga: Aljibe, 1999.

RUIZ, José. Ignacio. Metodología de la investigación cualitativa. Bilbao: Universidad de Deusto, 2003.

STRAUSS, Anselm; CORBIN, Juliet. Bases de la investigación cualitativa: Técnicas y procedimientos para desarrollar la teoría fundamentada. Colombia: Universidad de Antioquia, 2002. 
TORO, Sergio. Una aproximación epistemológica a la didáctica de la motricidad desde el discurso y práctica docente. Revista Estudios Pedagógicos, Valdivia, v.. 33, n.1, p. 29-43, 2007.

VALLES, Miguel. Técnicas cualitativas de investigación social: Reflexión metodológica y práctica profesional. Madrid: Síntesis, 2007.

Varias ideas del marco teórico de este trabajo y desde donde se realizan los análisis propuestos surgen en el contexto del Proyecto de Investigación financiado por el Fondo Nacional de Investigación Científica y Tecnológica de Chile (Fondecyt) № 11110016.

Endereço para correspondência:

Alberto Moreno Doña

Universidad Austral de Chile

Facultad de Filosofía y Humanidades

Instituto de Filosofía y Estudios Educacionales

Campus Isla Teja, $\mathrm{S} / \mathrm{N}$

C.P.: 5090000

Valdivia, Región de Los Ríos

Chile.

Recebido em: 05.04.2012

Aprovado em: 08.08.2012 
